\title{
ON SOME CLASSES OF ALMOST CONTACT METRIC MANIFOLDS
}

\author{
By \\ Jong Taek CHO*
}

\section{Introduction}

In [1] J. Berndt and L. Vanhecke introduced two classes ( $\$$ - and $\mathfrak{B}$-spaces) of Riemannian manifolds which include the class of locally symmetric spaces using the properties of Jaoobi operators along geodesics. They provided some characterizations of $\mathbb{(}$ - and $\mathfrak{B}$-spaces and gave the classifications for dimensions two and three. For further developments on the two spaces, we refer to [2], [3] and [8]. Further, T. Takahashi $([19])$ introduced the notion of a (Sasakian) locally $\varphi$-symmetric space which may be considered as the analogue in the almost contact metric case of locally Hermitian symmetric spaces. Also he gave examples and equivalent properties of Sasakian locally $\varphi$-symmetric spaces. For further results about the Sasakian locally $\varphi$-symmetric spaces, we refer to [5], [6].

In the present paper, we introduce in an analogous way as in [1] four classes of almost contact metric manifolds involving Sasakian locally $\varphi$-symmetric spaces. In section 2 , we recall definitions and several elementary properties of an almost contact, a contact, a $K$-contact metric manifold and a Sasakian manifold. In sections 3 and 4 we give the definitions of a DS -space, a DP-space, a $\xi(5$-space and a $\xi \Re$-space which are almost contact metric analogues of a $\mathbb{5}$-space or a $\mathfrak{B}$-space in the Riemannian case. We may observe that a Sasakian manifold is a $\xi \mathfrak{E}$-space and at the same time a $\xi \Re$-space. Also we prove that a Sasakian manifold is locally $\varphi$-symmetric if and only if it is a $\mathscr{D}($ s-space and at the same time a DP-space. In section 5, we show that the tangent sphere bundle of a 2-dimensional Riemannian manifold is a $\xi \Re$-space if and only if the base manifold is flat or of constant curvature 1. Furthermore, we give some examples of almost contact metric $\mathfrak{D}(5$-spaces and $\mathfrak{D P}$-spaces. In section 6 , we consider real hypersurfaces of a complex projective space $C P^{n}$ with FubiniStudy metric and determine $\xi \mathfrak{P}$-hypersurfaces of $\boldsymbol{C} P^{n}$. We also show that a homogeneous real hypersurface of $C P^{n}$ is a $\xi(5$-space, and moreover, we give

Received May 31, 1993. Revised September 3, 1993.

*) Partially supported by TGRC-KOSEF- 
a characterization of homogeneous real hypersurfaces of two types which appeared in the classification given by R. Takagi ([18]). All manifolds in the present paper are assumed to be connected and of class $C^{\infty}$ unless otherwise specified.

The author wishes to express his gratitude to Prof. K. Sekigawa for his many valuable advices and constant encouragement and to the referee for his valuable comments.

\section{Preliminaries}

In the present section, we recall definitions and elementary properties of an almost contact, a contact, a $K$-contact metric, and a Sasakian manifold. We refer to [4] for more details. A $(2 n+1)$-dimensional differentiable manifold $M$ is called an almost contact manifold it it admits a $(1,1)$-tensor field $\varphi$, a vector field $\xi$ and a 1 -form $\eta$ satisfying

$$
\eta(\xi)=1 \text { and } \varphi^{2}=-I+\eta \otimes \xi
$$

where $I$ denotes the identity transformation. From [2.1) we get

$$
\varphi \xi=0 \text { and } \eta \bullet \varphi=0 .
$$

Moreover, it is easily observed that an almost contact manifold $M$ admits a Riemannian metric $g$ such that

$$
g(\varphi X, \varphi Y)=g(X, Y)-\eta(X) \eta(Y)
$$

for all vector fields $X$ and $Y$ tangent to $M$. Setting $Y=\xi$ in (2.3), we also see that $\eta(X)=g(X, \xi)$. A Riemannian manifold equipped with structure tensors $(\varphi, \xi, \eta, g)$ satisfying $(2.1)$ and $(2.3)$ is called an almost contact metric manifold and denoted by $(M, \varphi, \xi, \eta, g)$. For an almost contact metric manifold $M=$ $(M, \varphi, \xi, \eta, g)$, one may define an almost complex structure $J$ on $M \times \boldsymbol{R}$ by $J(X, f(d / d t))=(\varphi X-f \xi, \eta(X)(d / d t))$, where $X$ is tangent to $M, f$ is a function on $M \times \boldsymbol{R}$ and $t$ the coordinate on $\boldsymbol{R}$. If the almost complex structure $J$ is integrable, $M$ is said to be normal. The integrability condition for the almost complex structure $J$ is the vanishing of the tensor field $[\varphi, \varphi]+2 d \eta \otimes \xi$, where $[\varphi, \varphi]$ denotes the Nijenhuis torson of $\varphi$.

Also, for an almost contact metric manifold we define its fundamental 2form $\Phi$ by

$$
\Phi(X, Y)=g(X, \varphi Y) .
$$

If $\Phi=d \eta, M=(M, \varphi, \xi, \eta, g)$ is called a contact metric manifold. In particular, we have $\eta \wedge(d \eta)^{n} \neq 0$. If the characteristic vector field $\xi$ of a contact metric 
manifold $M$ is a Killing vector field with respect to $g$, then $M$ is called a $K$ contact metric manifold. We denote by $R$ the curvature tensor defined by $R(X, Y) Z=\nabla_{X}\left(\nabla_{Y} Z\right)-\nabla_{Y}\left(\nabla_{X} Z\right)-\nabla_{[X, Y]} Z$, where $\nabla$ is the Levi-Civita connection and $X, Y, Z$ are vector fields. It is known that the curvature tensor of a $K$ contact metric manifold satisfies

$$
R(X, \xi) \xi=X-\eta(X) \xi .
$$

A normal contact metric manifold is called a Sasakian manifold. We may see that the conditions of being normal and contact metric are equivalent to

$$
\left(\nabla_{X} \varphi\right) Y=g(X, Y) \xi-\eta(Y) X .
$$

We note that (2.5) implies

$$
\nabla_{X} \xi=-\varphi X,
$$

from which it follows that $\xi$ is a Killing vector field. The curvature tensor of a Sasakian manifold satisfies

$$
\begin{gathered}
R(X, Y) \xi=\eta(Y) X-\eta(X) Y, \\
R(X, \xi) Y=\eta(Y) X-g(X, Y) \xi .
\end{gathered}
$$

\section{3. $\mathfrak{D C}$-spaces and $\mathfrak{D ß}$-spaces}

In this section, we introduce two classes (DS - and DP-spaces) of almost contact metric manifolds which extend Sasakian locally $\varphi$-symmetric spaces. Let $M=(M, \varphi, \xi, \eta, g)$ be an almost contact metric manifold. Let $T$ be a tensor field of type $(1,2)$ defined by (cf. [17])

$$
T_{X} Y=-\frac{1}{2} \varphi\left(\nabla_{X} \varphi\right) Y-\frac{1}{2} \eta(Y) \nabla_{X} \xi-\eta(X) \varphi Y+\left(\nabla_{X} \eta\right)(Y) \xi,
$$

for all vector fields $X$ and $Y$. We define a linear connection on $M$ by

$$
\bar{\nabla}_{X} Y=\nabla_{X} Y+T_{X} Y \text {. }
$$

The linear connection $\bar{\nabla}$ has the torsion tensor $T_{X} Y-T_{Y} X$. Also, using (2.1) and (2.2), we have

$$
\bar{\nabla} \varphi=0, \quad \bar{\nabla} \xi=0, \quad \bar{\nabla} \eta=0, \quad \bar{\nabla} g=0 .
$$

We remark that the above connection $\bar{\nabla}$ coincides with the Tanaka connection (defined in [20]) on a strongly pseudo-convex integral $C R$-manifold whose structure is determined by a given contact metric structure (see Proposition 2.1 in [22]).

The tangent space $T_{p} M$ of $M$ at $p \in M$ decomposes as $T_{p} M=\mathscr{D}_{p} \oplus \xi_{p}$ (direct 
sum), where we denote $\mathfrak{D}_{p}=\left\{v \in T_{p} M \mid \eta(v)=0\right\}$. Then $\mathscr{D}: p \rightarrow \mathscr{D}_{p}$ defines a distribution orthogonal to $\xi$. From (3.2) we see that a $\bar{\nabla}$-geodesic (not necessarily a $(\nabla$-)geodesic) which is initially tangent to $\mathscr{D}$ remains tangent to $\mathscr{D}$, where a $\bar{\nabla}$-geodesic means a geodesic with respect to the linear connection $\bar{\nabla}$. We call such a $\bar{\nabla}$-geodesic which is tangent to $\mathfrak{D}$ a horizontal $\bar{\nabla}$-geodesic. Let $\gamma$ be a horizontal $\bar{\nabla}$-geodesic parametrized by the arc-length parameter $s$. We denote $\dot{\gamma}=\gamma_{*}(d / d s)$ where $\gamma_{*}$ is the differential of $\gamma: I \rightarrow M$. Using the Jacobi operator $R_{\dot{\gamma}}=R(\cdot, \dot{\gamma}) \dot{\gamma}$ along $\gamma$, we introduce two new classes $\mathfrak{D C}$ and $\mathfrak{D P}$ of almost contact metric manifolds as analogous concepts of the $(5$ - and $\mathfrak{B}$-classes (defined in [1]) of Riemannian manifolds. Namely, we denote by $\mathfrak{D C}$ the class of almost contact metric manifolds such that the eigenvalues of $R_{\dot{\gamma}}$ are constant along $\gamma$ and by $\mathfrak{D P}$ that of almost contact metric manifolds such that $R_{\dot{\gamma}}$ is diagonalizable by a parallel orthonormal frame field along $\gamma$ with respect to $\bar{\nabla}$, for any $\bar{\nabla}$-geodesic $\gamma$ whose tangent vectors belong to $\mathfrak{D}$. An almost contact metric manifold $M$ is said to be a $\mathfrak{D C}$-space (resp. DB-space) if $M$ belongs to $\mathfrak{D C}$ (resp. $\mathfrak{D P})$.

In particular, let $M=(M, \varphi, \xi, \eta, g)$ be a Sasakian manifold. Then by (2.5) and (2.6) we have

$$
T_{X} Y=g(X, \varphi Y) \xi-\eta(X) \varphi Y+\eta(Y) \varphi X
$$

for all vector fields $X$ and $Y$ on $M$. Moreover, we have $T_{X} X=0$ and

$$
\bar{\nabla} \varphi=0, \quad \bar{\nabla} \xi=0, \quad \bar{\nabla}_{\eta}=0, \quad \bar{\nabla} g=0, \quad \bar{\nabla} T=0 .
$$

Also, we have

$$
\begin{aligned}
\left(\bar{\nabla}_{V} R\right)(X, Y) Z= & \left(\nabla_{V} R\right)(X, Y) Z+g(V, \varphi R(X, Y) Z) \xi-\eta(V) \varphi R(X, Y) Z \\
& +\eta(R(X, Y) Z) \varphi V-g(V, \varphi X) R(\xi, Y) Z+\eta(V) R(\varphi X, Y) Z \\
& -\eta(X) R(\varphi V, Y) Z-g(V, \varphi Y) R(X, \xi) Z+\eta(V) R(X, \varphi Y) Z \\
& -\eta(Y) R(X, \varphi V) Z-g(V, \varphi Z) R(X, Y) \xi+\eta(V) R(X, Y) \varphi Z \\
& -\eta(Z) R(X, Y) \varphi V
\end{aligned}
$$

for all vector fields $V, X, Y, Z$ on $M$. From (3.4), using (2.7) and (2.8) we have

$$
\begin{gathered}
g\left(\left(\bar{\nabla}_{V} R\right)(X, Y) Z, \xi\right)=0, \\
g\left(\left(\bar{\nabla}_{V} R\right)(X, Y) Z, W\right)=g\left(\left(\nabla_{V} R\right)(X, Y) Z, W\right)
\end{gathered}
$$

for all $V, X, Y, Z, W \in \mathfrak{D}$. Taking account of the fact $T_{X} X=0$ and from (3.3), we have 
LEMMA 3.1. Let $M$ be a Sasakian manifold. Then a $\bar{\nabla}$-geodesic coincides with a $(\nabla$-)geodesic, and a geodesic which is initially tangent to $\mathfrak{D}$ remains tangent to $\mathfrak{D}$.

We recall the definition of a Sasakian locally $\varphi$-symmetric space ([19]).

Definition 3.2. A Sasakian manifold $M=(M, \varphi, \xi, \eta, g)$ is said to be a locally $\varphi$-symmetric space if the curvature tensor $R$ satisfies $\varphi^{2}\left(\nabla_{V} R\right)(X, Y) Z=0$ for all $V, X, Y, Z \in \mathfrak{D}$.

Taking account of $(2.1)$, we see that the condition $\varphi^{2}\left(\nabla_{V} R\right)(X, Y) Z=0$ is equivalent to $g\left(\left(\nabla_{V} R\right)(X, Y) Z, W\right)=0$ for all $V, X, Y, Z, W \in \mathfrak{D}$.

Now we give a characterization of a Sasakian locally $\varphi$-symmetric space.

THEOREM 3.3. Let $M$ be a Sasakian manifold. Then $M$ is locally $\varphi$-symmetric if and only if $M$ belongs to $\mathfrak{D} \cap \mathfrak{D P}$, i.e., $M$ is $a \mathfrak{D}($-space and at the same time a Dß-space.

Proof. Let $M$ be a locally $\varphi$-symmetric space and $\gamma: I \rightarrow M$ be a geocesic parametrized by the arc-length parameter $s$ with $\dot{\gamma}(0) \in \mathscr{D}_{r(0)}$. Then from Lemma 3.1 we see that $\gamma$ is also a $\bar{\nabla}$-geodesic and $\dot{\gamma}(s) \in \mathfrak{D}$ for all $s \in I$. At first, for the vector field $\xi$, we see that $\bar{\nabla}_{\dot{\gamma}} \xi=0$ and $R_{\dot{\gamma}} \xi=\xi$ from $(2.8)$. Thus it is sufficient to consider the Jacobi operator $R_{\dot{\gamma}}$ on $\mathscr{D}$. Now we assume $R_{\dot{\gamma}}\left(s_{0}\right) v=\kappa v$ for some $s_{0} \in I$ and $v \in \mathfrak{D}_{\gamma\left(s_{0}\right)}$. Let $E_{v}$ be the parallel vector field with respect to $\bar{\nabla}$ along $\gamma$ with $E_{v}\left(s_{0}\right)=v$. Then since $M$ is locally $\varphi$-symmetric, from (3.5) and (3.6) we see that $R_{\dot{\gamma}} E_{v}$ and $\kappa E_{v}$ are parallel vector fields alongs $\gamma$ with respect to $\bar{\nabla}$. Thus we have $R_{\dot{\gamma}} E_{v}=\kappa E_{v}$. Therefore we have the conclusion.

Conversely, let us assume that $M$ is a $\mathscr{D}(5$-space and at the same time a DP-space. Then by definition we may assume that $R_{\dot{\gamma}} E_{i}=\kappa_{i} E_{i}, i=1,2, \cdots$, $2 n+1$, where $\kappa_{i}$ are constant along $\gamma$ and $\left\{E_{i}\right\}$ is an orthonormal parallel frame field along $\gamma$ with respect to $\bar{\nabla}$. By covariantly differentiating both sides of the above equations with respect to $\bar{\nabla}$ along $\gamma$ (as a $\bar{\nabla}$-geodesic), we get $\left(\bar{\nabla}_{\dot{\gamma}} R\right.$ ) $(\cdot, \dot{\gamma}) \dot{\gamma}=0$, which implies $\left(\bar{\nabla}_{v} R\right)(\cdot, v) v=0$ for any $v \in D_{p}$ and $p \in M$. Thus with (3.6) we have $g\left(\left(\bar{\nabla}_{V} R\right)(X, V) V, W\right)=g\left(\left(\nabla_{V} R\right)(X, V) V, W\right)=0$ for all $V, X, W \in \mathfrak{D}$. By polarization of the above equation and using the first and the second Bianchi identities, we have $g\left(\left(\nabla_{V} R\right)(X, Y) Z, W\right)=0$ for all $V, X, Y, Z, W \in \mathfrak{D}$ (cf. [9], [23]). Therefore from Definition 3.2 we see that $M$ is locally $\varphi$-symmetric.

(Q.E. D.) 
REMARK 3.4. In particular, let $M$ be a 3-dimensional Sasakian manifold. It is well-known that the curvature tensor $R$ of a 3-dimensional Riemannian manifold is expressed by

$$
\begin{aligned}
R(X, Y) Z= & \rho(Y, Z) X-\rho(X, Z) Y+g(Y, Z) Q X-g(X, Z) Q Y \\
& -\frac{1}{2} \tau\{g(Y, Z) X-g(X, Z) Y\}
\end{aligned}
$$

for all vector fields $X, Y, Z$, where $Q$ is the Ricci $(1,1)$-tensor determined by $\rho(X, Y)=g(Q X, Y)$ and $\tau$ is the scalar curvature of the manifold. Let $\gamma$ be a geodesic parametrized by the arc-length parameter $s$ with $\dot{\gamma}(s) \in \mathscr{D}_{\gamma(s)}$ (see Lemma 3.1). From (3.3) we see that $\{\dot{\gamma}, \varphi \dot{\gamma}, \xi\}$ is a parallel orthonormal frame field along $\gamma$ with respect to $\bar{\nabla}$. From $(2.8)$ and $(3.7)$, we have $R(\xi, \dot{\gamma}) \dot{\gamma}=R(\xi, \varphi \dot{\gamma}) \varphi \dot{\gamma}$ $=\xi$ and $R(\varphi \dot{\gamma}, \dot{\gamma}) \dot{\gamma}=\{(1 / 2) \tau-\rho(\xi, \xi)\} \varphi \dot{\gamma}$. Thus we see that a 3-dimensional Sasakian manifold is a DPS-space. Applying Theorem 3.3 to the 3-dimensional case, we see that a 3-dimensional Sasakian manifold is locally $\varphi$-symmetric if and only if the scalar curvature is constant for all directions orthogonal to $\xi$. This gives another proof of Theorem 4.1 in [24].

Returning to the general case, we characterize an almost contact metric $\mathfrak{D C}$-space and $\mathfrak{D P}$-space in a similar way as in $[1]$. We prove

Proposition 3.5. An almost contact metric manifold $M$ is a D(s-space if and only if for each $p \in M$ and $v \in \mathfrak{D}_{p}$, there exists an endomorphism $S_{v}$ of $T_{p} M$ such that $R_{v}^{\prime}=R_{v^{\circ}} S_{v}-S_{v^{\circ}} R_{v}$ where we denote $R_{v}^{\prime}=\left(\bar{\nabla}_{v} R\right)(\cdot, v) v$.

Proof. Let $M$ be a $\mathfrak{D C}$-space and $\gamma$ be a horizontal $\bar{\nabla}$-geodesic in $M$ which is parametrized by the arc-length parameter $s$ and $\gamma(0)=p$ and $\dot{\gamma}(0)=v$ for any $p \in M$ and $v \in \mathscr{D}_{p}$. Let $\tau_{0, s}^{\gamma}$ be the parallel translation along $\gamma$ from $\gamma(0)$ to $\gamma(s)$ with respect to $\bar{\nabla}$. Then from the property $\bar{\nabla} g=0$, we see that $\tau^{\gamma}$ is an isometry along $\gamma$. Now we put $A(s)=\tau_{s, 0^{\gamma}}^{\gamma} R_{\dot{\gamma}^{\circ}} \tau_{0, s}^{\gamma}$, then $A(s)$ is a family of selfadjoint endomorphisms of $T_{p} M$ and the eigenvalues of $A(s)$ are constant. Thus applying Lemma 4 in [1], there exists a family of endomorphisms $S(s)$ of $T_{p} M$ such that $A^{\prime}(s)=A(s) \circ S(s)-S(s) \circ A(s)$. This implies $A^{\prime}(0)=A(0) \circ S(0)-S(0) \circ A(0)$. Thus we have $R_{\dot{\gamma}}^{\prime}(0)=R_{\dot{\gamma}}(0) \circ S(0)-S(0) \circ R_{\dot{\gamma}}(0)$, and hence $R_{v}^{\prime}=R_{v} \circ S_{v}-S_{v} \circ R_{v}$ where $S_{v}=S(0)$. In order to prove the converse, let $\gamma: I \rightarrow M$ be a horizontal $\bar{\nabla}$-geodesic parametrized by the arc-length parameter $s$ with $\gamma\left(s_{0}\right)=p, s_{0} \in I$. Let $A(s)=$ $\tau_{s, s_{0}}^{\gamma} \circ R_{\dot{\gamma}}(s) \circ \tau_{s_{0}, s}^{\gamma}$ and $S(s)=\tau_{s_{,} s_{0}}^{\gamma} \circ S_{\dot{\gamma}(s)} \circ \tau_{s_{0}, s}^{\gamma}$. Then we see that $A(s)$ and $S(s)$ are families of endomorphisms of $T_{p} M$ and by a calculation we have 


$$
\begin{aligned}
A^{\prime}(s) & =\tau_{s, s_{0}}^{\gamma} \circ R_{\dot{\gamma}}^{\prime} \circ \tau_{s_{0}, s}^{\gamma} \\
& =\tau_{s, s_{0}}^{\gamma} \circ\left(R_{\dot{\gamma}} \circ S_{\dot{\gamma}}-S_{\dot{\gamma}} \circ R_{\dot{\gamma}}\right) \circ \tau_{s_{0}, s}^{\gamma}(\text { by the assumption) } \\
& =A(s) \circ S(s)-S(s) \circ A(s),
\end{aligned}
$$

i. e., there exists a family of endomorphisms $S(s)$ of $T_{p} M$ such that $A^{\prime}(s)=A(s)$ $\circ S(s)-S(s) \circ A(s)$. Thus by Lemma 4 in [1], we see that the eigenvalues of the endomorphism $A$, and therefore also of $R_{\dot{\gamma}}$ are constant. (Q.E.D.)

On the other hand, as a characterization of an almost contact metric $\mathfrak{D P}$ space, we have

Proposition 3.6. If $M$ is a Dßs-space, then $R_{v} \circ R_{v}^{\prime}=R_{v}^{\prime} \circ R_{v}$ for all $v \in \mathscr{D}_{p}$, $p \in M$, where $R_{v}^{\prime}=\left(\bar{\nabla}_{v} R\right)(\cdot, v) v$. Moreover, if $M$ is real analytic, then also the converse holds.

We refer to Lemma 5 in [1] for the proof of the above Proposition 3.6.

\section{4. $\xi \llbracket$-spaces and $\xi \Re$-spaces}

In this section, we study local symmetry in the direction $\xi$. All almost contact metric manifolds do not satisfy the following condition: (*) each trajectory of $\xi$ is a geodesic. However some special cases of almost contact metric manifold do satisfy it. For example, the tangent sphere bundle of a Riemannian manifold as a hypersurface of the tangent bundle with an almost Kähler structure inherits an almost contact metric structure and satisfies $(*)$ (cf. chapter 7 in [4]). Another example is a homogeneous real hypersurface of an $n$-dimensional complex projective space $\boldsymbol{C} P^{n}$ with Fubini-Study metric (cf. [11]). We may also observe that every contact metric manifold satisfies the condition (*) (cf. [4]). Moreover, from (2.4) and (2.7), we see that a $K$-contact metric manifold and a Sasakian manifold satisfy in addition $\left(\nabla_{\xi} R\right)(\cdot, \xi) \xi=0$.

Definition 4.1. An almost contact metric manifold $M$ with a structure $(\varphi, \xi, \eta, g)$ is said to be a locally $\xi$-symmetric space if $M$ satisfies (*) (i.e., $\nabla_{\xi} \xi$ $=0)$ and $\left(\nabla_{\xi} R\right)(\cdot, \xi) \xi=0$.

We remark that a contact metric manifold whose characteristic vector field $\xi$ belongs to the $k$-nullity distribution (see [21]) is a locally $\xi$-symmetric space. We may characterize a locally $\xi$-symmetric space using the Jacobi operator $R_{\xi}=R(\cdot, \xi) \xi$ associated with the vector field $\xi$ in a similar way as in Theorem 1 in [1]. Namely, an almost contact metric manifold $M$ satisfying the condi- 
tion (*) is locally $\xi$-symmetric if and only if $M$ satisfies the following two conditions: (c) the eigenvalues of $R_{\xi}$ are constant along each trajectory of $\xi$ and $(p) R_{\xi}$ is diagonalizable by a parallel orthonormal frame field along each trajectory of $\xi$. We denote by $\xi(5$ the class of almost contact metric manifolds with $(*)$ and $(c)$, and by $\xi \Re$ that of almost contact metric manifolds with $(*)$ and $(p)$. An almost contact metric manifold $M$ is said to be a $\xi(5$-space (resp. $\xi \mathfrak{P}$-space) if $M$ belongs to $\xi \Subset$ (resp. $\xi \Re$ ).

From Theorem 2 (resp. Theorem 5) in [1], we immediately have the following Remark 4.2 (resp. Remark 4.3) as a characterization of a $\xi($-(resp. $\xi \Re$-) space.

REMARK 4.2. An almost contact metric manifold $M$ is a $\xi \S$-space if and only if $M$ satisfies $(*)$ and there exists a skew-symmetric $(1,1)$-tensor field $B_{\xi}$ such that $\dot{R}_{\xi}=R_{\xi} \circ B_{\xi}-B_{\xi} \circ R_{\xi}$ where we denote $\dot{R}_{\xi}=\left(\nabla_{\xi} R\right)(\cdot, \xi) \xi$.

REMARK 4.3. If an almost contact metric manifold $M$ is a $\xi \Re$-space, then we have $R_{\xi} \circ \dot{R}_{\xi}=\dot{R}_{\xi} \circ R_{\xi}$ and moreover, if $M$ satisfies (*) and is real analytic, then the converse holds.

Also, we have some interesting equivalent properties of a $\xi \mathfrak{B}$-space related to the geometry of Jacobi vector fields and the geometry of geodesic spheres along geodesic trajectories of $\xi$. For more details concerning that, we refer to [1] and [2].

\section{Tangent sphere bundle of a surface}

Let $M$ be a 2-dimensional Riemannian manifold and $T_{1} M$ the tangent sphere bundle of $M$ (i.e., the set of all unit tangent vectors of $M$ ) with the projection map $\pi: T_{1} M \rightarrow M$. As we stated in the first part of section 4 , it is known that the tangent bundle $T M$ admits an almost Kähler structure $(J, \bar{g})$ (cf. chapter 7 in [4]). Let $\left(x^{1}, x^{2}\right)$ be an isothermal local coordinate system on $M$ such that the Riemannian metric is of the form

$$
\rho^{2}\left(\left(d x^{1}\right)^{2}+\left(d x^{2}\right)^{2}\right)
$$

where $\rho$ is a function on $M$. Then by a calculation we see that the Gauss curvature $\kappa$ of $M$ is $-\left(\Delta_{0} \log \rho / \rho^{2}\right)$ where $\Delta_{0}$ is the Laplacian with respect to Euclidean metric. Let $\left(u^{1}, u^{2}, y^{1}, y^{2}\right)$ be a local coordinate system around a point $p$ of $T_{1} M$ in $T M$ sucn that $u^{i}=x^{i} \circ \pi$ and $\rho^{2}\left(\left(y^{1}\right)^{2}+\left(y^{2}\right)^{2}\right)=1$. The vector field $N=y^{1}\left(\partial / \partial y^{1}\right)+y^{2}\left(\partial / \partial y^{2}\right)$ is a unit normal and the position vector for the point $p$ of $T_{1} M$. Denote by $g$ the metric of $T_{1} M$ induced from $\bar{g}$ on $T M$. 
Define $\varphi, \xi, \eta$ by

$$
J N=-\xi, \quad J X=\varphi X+\eta(X) N .
$$

Then we see that $(\varphi, \xi, \eta, g)$ is an almost contact metric structure of $T_{1} M$ and we have a local orthonormal frame field $\left\{e_{1}, e_{2}, e_{3}\right\}$ as follows:

$$
\begin{aligned}
& e_{3}=\xi=\sum_{i j k}\left(y^{i} \frac{\partial}{\partial u^{i}}-\left\{\begin{array}{cc}
i & \\
j & k
\end{array}\right\} y^{j} y^{k} \frac{\partial}{\partial y^{i}}\right) . \\
& e_{1}=\sum_{i} z^{i} \frac{\partial}{\partial y^{i}}, \\
& e_{2}=-\varphi e_{1}=\sum_{i j k}\left(z^{i} \frac{\partial}{\partial u^{i}}-\left\{\begin{array}{ll}
i & \\
j & k
\end{array}\right\} y^{j} z^{k} \frac{\partial}{\partial y^{i}}\right)
\end{aligned}
$$

for $i, j, k=1,2$ where we denote $\left(z^{1}, z^{2}\right)=\left(-y^{2}, y^{1}\right),\left\{\begin{array}{cc}i \\ j & k\end{array}\right\}=\left\{\begin{array}{c}i \\ j\end{array}\right\} \circ \pi$ and where $\left\{\begin{array}{cc}i \\ j & k\end{array}\right\}$ are the Christoffel symbols of the Riemannian connection of $M$.

For the local orthonormal frame field we have

$$
\left[e_{1}, e_{2}\right]=-e_{3}, \quad\left[e_{2}, e_{3}\right]=-\tilde{\kappa} e_{1}, \quad\left[e_{3}, e_{1}\right]=-e_{2},
$$

where $\tilde{\kappa}=\kappa \circ \pi$. Put

$$
\Gamma_{i j k}=g\left(\nabla_{e_{i}} e_{j}, e_{k}\right) \quad \text { for } i, j, k=1,2,3 .
$$

Then we have $\Gamma_{i j k}=-\Gamma_{i k j}$. We recall the formula

$$
\begin{aligned}
2 g\left(\nabla_{X} Y, Z\right)= & X g(Y, Z)+Y g(Z, X)-Z g(X, Y)+g(Y,[Z, X]) \\
& +g(Z,[X, Y])-g(X,[Y, Z])
\end{aligned}
$$

for all vector fields $X, Y, Z$ on $T_{1} M$. Using this formula, we obtain

$$
\Gamma_{123}=\frac{1}{2}(\tilde{\kappa}-2), \quad \Gamma_{213}=\Gamma_{321}=\frac{\tilde{\kappa}}{2}, \quad \text { all other } \Gamma_{i j k} \text { being zero. }
$$

From (5.3) we see that $e_{1}, e_{2}, e_{3}$ are all geodesic vector fields, i.e., self-parallel vector fields and from (5.2) and (5.3) we get

$$
\begin{aligned}
& R\left(e_{1}, e_{3}\right) e_{3}=\frac{1}{4} \tilde{\kappa}^{2} e_{1}+\frac{1}{2}\left(e_{3} \tilde{\kappa}\right) e_{2}, \\
& R\left(e_{2}, e_{3}\right) e_{3}=\frac{1}{2}\left(e_{3} \tilde{\kappa}\right) e_{1}-\left(\frac{3}{4} \tilde{\kappa}_{2}-\tilde{\kappa}\right) e_{2}, \\
& R\left(e_{2}, e_{1}\right) e_{1}=\frac{1}{4} \tilde{\kappa}^{2} e_{2}, \\
& R\left(e_{3}, e_{1}\right) e_{1}=\frac{1}{4} \tilde{\kappa}^{2} e_{3},
\end{aligned}
$$




$$
\begin{aligned}
& R\left(e_{1}, e_{2}\right) e_{2}=\frac{1}{4} \tilde{\kappa}^{2} e_{1}-\frac{1}{2}\left(e_{2} \tilde{\kappa}\right) e_{3}, \\
& R\left(e_{3}, e_{2}\right) e_{2}=-\frac{1}{2}\left(e_{2} \tilde{\kappa}\right) e_{1}-\left(\frac{3}{4} \tilde{\kappa}^{2}-\tilde{\kappa}\right) e_{3} .
\end{aligned}
$$

Moreover, we have

$$
\begin{aligned}
& \left(\nabla_{e_{3}} R\right)\left(e_{1}, e_{3}\right) e_{3}=\tilde{\kappa}\left(e_{3} \tilde{\kappa}\right) e_{1}+\frac{1}{2}\left\{e_{3}\left(e_{3} \tilde{\kappa}\right)-\tilde{\kappa}^{3}+\tilde{\kappa}^{2}\right\} e_{2} \\
& \left(\nabla_{e_{3}} R\right)\left(e_{2}, e_{3}\right) e_{3}=\frac{1}{2}\left\{e_{3}\left(e_{3} \tilde{\kappa}\right)-\tilde{\kappa}^{3}+\tilde{\kappa}^{2}\right\} e_{1}+\left\{e_{3} \tilde{\kappa}-2 \tilde{\kappa}\left(e_{3} \tilde{\kappa}\right)\right\} e_{2}
\end{aligned}
$$

PROPOSITION 5.1. The tangent sphere bundle $T_{1} M$ of a 2-dimensional Riemannian manifold $M$ is a $\xi$ (5-space if and only if the Gauss curvature of $M$ is constant.

PROOF. From (5.4) we have the following matrix representation of $R_{\xi}$ with respect to $\left\{e_{1}, e_{2}, e_{3}\right\}$ :

$$
R_{\xi}=\left(\begin{array}{lcc}
\frac{1}{4} \tilde{\kappa}^{2} & \frac{1}{2}\left(e_{3} \tilde{\kappa}\right) & 0 \\
\frac{1}{2}\left(e_{3} \tilde{\kappa}\right) & -\frac{3}{4} \tilde{\kappa}^{2}+\tilde{\kappa} & 0 \\
0 & 0 & 0
\end{array}\right) .
$$

The eigenvalues $\lambda_{i}, i=1,2,\left(\lambda_{3}=0\right)$ of $R_{\xi}$ are

$$
\begin{aligned}
& \lambda_{1}=\frac{-\frac{1}{2}-\tilde{\kappa}^{2}+\tilde{\kappa}+\sqrt{\tilde{\kappa}^{2}(\tilde{\kappa}-1)^{2}+\left(e_{3} \tilde{\kappa}\right)^{2}}}{2} \\
& \lambda_{2}=-\frac{1}{2} \tilde{\kappa}^{2}+\tilde{\kappa}-\sqrt{\tilde{\kappa}^{2}(\tilde{\kappa}-1)^{2}+\left(e_{3} \tilde{\kappa}\right)^{2}} \\
& 2
\end{aligned} .
$$

Now we assume that the tangent sphere bundle $T_{1} M$ of a 2-dimensional Riemannian manifold $M$ is a $\xi$ (5-space, that is, the eigenvalues $\lambda_{i}(i=1,2)$ of $R_{\xi}$ are constant along each trajectory of $\xi$. Let $W=\left\{p \in T_{1} M \mid \lambda_{1}(p) \neq \lambda_{2}(p)\right\}$. Then $W$ is an open and dense subset of $T_{1} M$. Thus we have $\xi\left(\lambda_{1}+\lambda_{2}\right)=0$ on $W$, which implies that $\xi \tilde{\kappa}=0$ on $W$. From the continuity of $\tilde{\kappa}$, we see that $\xi \tilde{\kappa}=0$ on $T_{1} M$ and from $(5.1)$ we conclude that $\kappa$ is constant on $M$. Conversely, if $\kappa$ is constant on $M$, then $\tilde{\kappa}=\kappa \circ \pi$ is also constant on $T_{1} M$. Thus, from (5.4) and (5.6), we have 


$$
R_{\xi}=\left(\begin{array}{ccc}
\frac{1}{4} \tilde{\kappa}^{2} & 0 & 0 \\
0 & -\frac{3}{4} \tilde{\kappa}^{2}+\tilde{\kappa} & 0 \\
0 & 0 & 0
\end{array}\right) \text { and } \dot{R}_{\nu}=\left(\begin{array}{ccc}
0 & -\frac{1}{2} \tilde{\kappa}^{3}+\frac{1}{2} \tilde{\kappa}^{2} & 0 \\
-\frac{1}{2} \tilde{\kappa}^{3}+\frac{1}{2} \tilde{\kappa}^{2} & 0 & 0 \\
0 & 0 & 0
\end{array}\right)
$$

with respect to $\left\{e_{1}, e_{2}, e_{3}\right\}$. Put

$$
B_{\xi}=\left(\begin{array}{ccc}
0 & -\frac{1}{2} \tilde{\kappa} & 0 \\
\frac{1}{2} \tilde{\kappa} & 0 & 0 \\
0 & 0 & 0
\end{array}\right)
$$

Then we have $\dot{R}_{\xi}=R_{\xi^{\circ}} B_{\xi}-B_{\xi^{\circ}} R_{\xi}$. Thus from Remark 4.2 we see that the tangent sphere bundle $T_{1} M$ is a $\xi(5$-space. (Q.E.D.)

THEOREM 5.2. The tangent sphere bundle $T_{1} M$ of a 2-dimensional Riemannian manifold $M$ is a $\xi \mathfrak{P}$-space (or locally $\xi$-symmetric space) if and only if the Gauss curvature of $M$ is 0 or 1 .

ProOF. Assume that $T_{1} M$ is a $\xi \Re$-space. Then from Remark 4.3 we see that $T_{1} M$ satisfies $R_{\xi} \circ \dot{R}_{\xi}=\dot{R}_{\xi} \circ R_{\xi}$, where $\dot{R}_{\xi}=\left(\nabla_{\xi} R\right)(\cdot, \xi) \xi$. From (5.4) and (5.6), we calculate $R_{\xi}\left(\dot{R}_{\xi}\left(e_{i}\right)\right)=\dot{R}_{\xi}\left(R_{\xi}\left(e_{i}\right)\right)$ for $i=1,2$. Then we have

$$
\tilde{\kappa}^{5}-2 \tilde{\kappa}^{4}+\tilde{\kappa}^{3}-(\xi(\xi \tilde{\kappa})) \tilde{\kappa}^{2}+\left\{3(\xi \tilde{\kappa})^{2}+\xi(\xi \tilde{\kappa})\right\} \tilde{\kappa}-(\xi \tilde{\kappa})^{2}=0 .
$$

From the above equation, we have $\tilde{\kappa}^{5}-2 \tilde{\kappa}^{4}+\tilde{\kappa}^{3}=\tilde{\kappa}^{3}\left(\tilde{\kappa}^{2}-2 \kappa+1\right)=0$. Thus we see that $\kappa=0$ or 1 . Conversely, if $\kappa=0$ or 1 , then from (5.4) we see that $T_{1} M$ is flat or a space of constant sectional curvature $1 / 4$. Thus we see that $T_{1} M$ is of course a $\xi \Re$-space. We recall that a locally $\xi$-symmetric space is equivalently characterized as a $\xi \mathcal{\xi}$ - which is at the same time a $\xi \mathfrak{B}$-space. Thus from the result of Proposition 5.1 we see that $T_{1} M$ is a $\xi \Re$-space if and only if it is a locally $\xi$-symmetric space. (Q.E.D.)

We remark that ([13]) $T_{1}\left(S^{2}\right)$ is isometric to the elliptic space $\boldsymbol{R} P^{3}$ of constant curvature $1 / 4$, where $S^{2}$ is the unit sphere in a Euclidean space $\boldsymbol{E}^{3}$ with the induced metric.

On the other hand, from (3.1), (3.2) and (5.3) we have

$$
\bar{\nabla}_{e_{i}} \xi=0 \text { and } \quad \bar{\nabla}_{e_{i}} e_{j}=0 \quad \text { for } i, j=1,2
$$

and moreover, we have 


$$
\begin{aligned}
& \left(\bar{\nabla}_{e_{1}} R\right)\left(e_{2}, e_{1}\right) e_{1}=0 \\
& \left(\nabla_{e_{1}} R\right)\left(e_{3}, e_{1}\right) e_{1}=0 \\
& \left(\nabla_{e_{2}} R\right)\left(e_{1}, e_{2}\right) e_{2}=\frac{1}{2} \tilde{\kappa}\left(e_{2} \tilde{\kappa}\right) e_{1}-\frac{1}{2} e_{2}\left(e_{2} \tilde{\kappa}\right) e_{3}, \\
& \left(\nabla_{e_{2}} R\right)\left(e_{3}, e_{2}\right) e_{2}=-\frac{1}{2} e_{2}\left(e_{2} \tilde{\kappa}\right) e_{1}-\frac{1}{2}\left\{3 \tilde{\kappa}\left(e_{2} \tilde{\kappa}\right)-2\left(e_{2} \tilde{\kappa}\right)\right\} e_{3} .
\end{aligned}
$$

Proposition 5.3. The tangent sphere bundle $T_{1} M$ of a 2-dimensional Riemannian manifold $M$ is a DSs,-space if and only if the Gauss curvature of $M$ is constant.

Proof. Assume that the tangent sphere bundle $T_{1} M$ of a 2-dimensional manifold $M$ is a $\mathfrak{D C}$-space. Using a similar calculation and argument as in the proof of Proposition 5.1, we see that $\kappa$ is constant on $M$. Conversely, we assume that $\kappa$ is constant on $M$. Taking an endomorphism $S_{v}=0$ of $T_{p}\left(T_{1} M\right)$ for any $v \in \mathscr{D}_{p}$ and $p \in T_{1} M$, then from (5.5), (5.8) and Proposition 3.5, we see that $T_{1} M$ is a $\mathscr{D}($-space. (Q.E. D.)

Proposition 5.4. The tangent sphere bundle $T_{1} M$ of a 2-dimensional Riemannian manifold is a DP-space if and only if the Gauss curvature of $M$ is constant.

Proof. Assume that $T_{1} M$ is a $\mathfrak{D P}$-space. Then from Proposition 3.6 we see that $T_{1} M$ satisfies $R_{v} \circ R_{v}^{\prime}=R_{v}^{\prime} \circ R_{v}$ for all $v \in \mathscr{D}_{p}, p \in T_{1} M$, where $R_{v}^{\prime}=\left(\bar{\nabla}_{v} R\right)$. $(\cdot, v) v$. From (5.5) and (5.8) we calculate $R_{e_{2}}\left(R_{e_{2}}^{\prime}\left(e_{a}\right)\right)=R_{e_{2}}^{\prime}\left(R_{e_{2}}\left(e_{a}\right)\right)$ for $a=1,3$. Then we get

$$
\left(e_{2} \tilde{\kappa}\right)^{2}(1-2 \tilde{\kappa})+\left(e_{2}\left(e_{2} \tilde{\kappa}\right)\right) \tilde{\kappa}(\tilde{\kappa}-1)=0 .
$$

From the above equation, we see that $\kappa$ is constant. Conversely, if $\kappa$ is constant, then with (5.8) taking account of (5.3) and (5.7), we have $\left(\nabla_{e_{i}} R\right)\left(\cdot, e_{\jmath}\right) e_{k}$ $=0$ for $i, j, k=1,2$. It may be observed that a $\mathscr{D}(5$ - which is at the same time a $\mathfrak{D P}$-space is equivalently characterized by $\left(\bar{\nabla}_{V} R\right)(\cdot, V) V=0$ for any $V \in \mathfrak{D}$. Thus we see that $T_{1} M$ is a $\mathfrak{D P}$-space. (Q.E.D.)

\section{Real hypersurfaces of $C P^{n}$}

Let $\left(C P^{n}, g, J\right)$ be an $n$-dimensional complex projective space with FubiniStudy metric $g$ of constant holomorphic sectional curvature 4 , and let $M$ be an oriented real hypersurface of $C P^{n}$. We denote by the same $g$ the induced 
metric on $M$. Let $N$ be a unit normal vector field of $M$ in $C P^{n}$. For any vector field $X$ tangent to $M$, we put

$$
J X=\varphi X+\eta(X) N, \quad J N=-\xi .
$$

Then we may see that the $\operatorname{structnre}(\varphi, \xi, \eta, g)$ is an almost contact metric structure on $M$. By $\tilde{\nabla}$ we denote the Riemannian connection on $C P^{n}$ and by $\nabla$ the one on $M$ determined by the induced metric. The the Gauss and Weingarten formulas are given respectively by

$$
\tilde{\nabla}_{X} Y=\nabla_{X} Y+g(A X, Y) N, \quad \tilde{\nabla}_{X} N=-A X
$$

for any vector field $X$ and $Y$ tangent to $M$, where $A$ is the shape operator of $M$ in $C P^{n}$. An eigenvector (resp. eigenvalue) of the shape operator $A$ is called a principal curvature vector (resp. principal curvature). Also we denote by $V_{\lambda}$ the eigenspace of $A$ associated with an eigenvalue $\lambda$. From the fact $\tilde{\nabla} J=0$ and (6.1), making use of the Gauss and Weingarten formulas, we have

$$
\nabla_{X} \xi=\varphi A X
$$

Let $R$ be the curvature tensor of $M$. Then we have following Gauss and Codazzi equations :

$$
\begin{gathered}
R(X, Y) Z=g(Y, Z) X-g(X, Z) Y+g(\varphi Y, Z) \varphi X-g(\varphi X, Z) \varphi Y \\
+2 g(X, \varphi Y) \varphi Z+g(A Y, Z) A X-g(A X, Z) A Y, \\
\left(\nabla_{X} A\right) Y-\left(\nabla_{Y} A\right) X=\eta(X) \varphi Y-\eta(Y) \varphi X+2 g(X, \varphi Y) \xi .
\end{gathered}
$$

From (6.2), we have

LEMMA 6.1. Each trajectory of $\xi$ is a geodesic if and only if $\xi$ is a principal curvature vector.

Typical examples of real hypersurfaces in $C P^{n}$ on which the trajectory of $\xi$ is a geodesic are homogeneous ones which are classified by R. Takai ([18]). T.E. Cecil and P. J. Ryan $([7])$ investigated real hypersurfaces of $\boldsymbol{C} P^{n}$ on which $\xi$ is a principal curvature vector. They showed that if $\xi$ is a principal curvature vector and the corresponding focal map has constant rank, then $M$ lies on a tube of constant radius over a certain Kähler submanifold. Making use of this notion and the result of R. Takagi's classification, M. Kimura ([11]) proved the following

THEOREM 6.2. Let $M$ be a real hypersurface of $C P^{n} . M$ has constant principal curvałures and $\xi$ is principal if and only if $M$ is locally isometric to a 
homogeneous real hypersurface i.e., a tube of radius $r$ over one of the following Kähler submanifolds:

$\left(\mathrm{A}_{1}\right)$ a hyperplane $C P^{n-1}$, where $0<r<\pi / 2$;

$\left(\mathrm{A}_{2}\right)$ a totally geodesic $C P^{k}(1 \leqq k \leqq n-2)$, where $0<r<\pi / 2$;

(B) a complex quadric $Q^{n-1}$, where $0<r<\pi / 4$;

(C) a $C P^{1} \times C P^{(n-1 / 2)}$, where $0<r<\pi / 4$ and $n(\geqq 5)$ is odd;

(D) a complex Grassmann $G_{2,5}(C)$, where $0<r<\pi / 4, n=9$;

(E) a Hermitian symmetric space $S O(10) / U(5)$, where $0<r<\pi / 4, n=15$.

We note that the number of distinct eigenvalues of the above real hypersurfaces is 2,3 or 5 , and the principal curvature $\alpha$ corresponding to the vector field $\xi$ is $2 \cot 2 r$ with multiplicity 1 . For more details, we refer to [11] and [18]. We only state two lemmas without proofs.

LEMMA 6.3 ([14]). If $\xi$ is principal curvature vector, then the corresponding principal curvature $\alpha$ is constant.

Lemma 6.4 ([14]). Assume $A \xi=\alpha \xi$. If $A X=\lambda X$ for $X \perp \xi$, then we have $A \varphi X=(\alpha \lambda+2 / 2 \lambda-\alpha) \varphi X$.

Now we give a characterization of real hypersurfaces of $C P^{n}$ in the class $\xi \Re$ introduced in section 4.

Proposition 6.5. Let $M^{2 n-1}$ be a $\xi \Re$-hypersurface of $C P^{n}$. Suppose $A \xi \neq 0$. Then $M$ is locally isometric to a homogeneous real hypersurface of type $\left(\mathrm{A}_{1}\right)$ or $\left(\mathrm{A}_{2}\right)$. Moreover, any real hypersurface of type $\left(\mathrm{A}_{1}\right)$ or $\left(\mathrm{A}_{2}\right)$ is a $\xi \Re$-space.

Proof. Assume $M$ is a $\xi \mathfrak{B}$-hypersurface of $C P^{n}$. We see from Lemma 6.1 that $\xi$ is a principal curvature vector and from Lemma 6.3 that the corresponding principal curvature $\alpha$ is constant. Thus from (6.3) we have

$$
R_{\xi} X=X+\alpha A X-\left(1+\alpha^{2}\right) \eta(X) \xi
$$

and

$$
\begin{aligned}
\dot{R}_{\xi} X & =\left(\nabla_{\xi} R\right)(X, \xi) \xi \\
& =\alpha\left(\nabla_{\xi} A\right) X
\end{aligned}
$$

for any $X$ tangent to $M$.

From Remark 4.3, we have 


$$
\begin{aligned}
0 & =\left(R_{\xi} \circ \dot{R}_{\xi}-\dot{R}_{\xi} \circ R_{\xi}\right) X \\
& =\alpha^{2}\left\{A\left(\nabla_{\xi} A\right) X-\left(\nabla_{\xi} A\right) A X\right\} .
\end{aligned}
$$

Since $\alpha \neq 0$ (the assumption), we have $A\left(\nabla_{\xi} A\right) X-\left(\nabla_{\xi} A\right) A X=0$, and hence taking account of Lemma 6.3, from (6.2), (6.4) and (6.7), we have

$$
0=\left(\alpha A \varphi A X-A^{2} \varphi A X+A \varphi X\right)-\left(\alpha \varphi A^{2} X-A \varphi A^{2} X+\varphi A X\right)
$$

for any $X \in \mathscr{D}$. Assume $X \in V_{\lambda}$. Then from Lemma 6.4 we have

$$
0=\left(\alpha \lambda-\lambda \frac{\alpha \lambda+2}{2 \lambda-\alpha}+1\right)\left(\frac{\alpha \lambda+2}{2 \lambda-\alpha}-\lambda\right) \varphi X \text {. }
$$

Thus we have

$$
\alpha \lambda-\lambda \frac{\alpha \lambda+2}{2 \lambda-\alpha}+1=0 \quad \text { or } \quad \frac{\alpha \lambda+2}{2 \lambda-\alpha}-\lambda=0,
$$

which implies $\lambda^{2}-\alpha \lambda-1=0 \quad(\alpha \neq 0)$, and hence $\lambda(2 \lambda-\alpha)=\alpha \lambda+2$, that is, $\lambda=$ $(\alpha \lambda+2 / 2 \lambda-\alpha)$. From this we conclude that $\varphi V_{\lambda}=V_{\lambda}$ and our real hypersurface $M$ must be locally isometric to one of real hypersurface of type $\left(\mathrm{A}_{1}\right)$ and $\left(\mathrm{A}_{2}\right)$ (cf. [16]). Taking account of the fact that every homogeneous manifold admits an analytic structure (refer to p. 123 in [10]), from the Remark 4.3 and (6.7), we see that any real hypersurface of type $\left(A_{1}\right)$ or $\left(A_{2}\right)$ is a $\xi \Re$-space. (Q.E.D.)

The above Proposition 6.3 is an improvement of the result obtained by $\mathrm{M}$. Kimura and S. Maeda ([12]). Also we remark that a homogeneous real hypersurface of type $\left(\mathrm{A}_{2}\right)$ is a locally $\xi$-symmetric space which is not a $K$-contact metric (and of course, not Sasakian) manifold. (cf. [15]).

We see from (6.5) that homogeneous real hypersurfaces of $C P^{n}$ are $\xi \Re$-spaces. Applying Remark 4.2, then from (6.5) and (6.6) we have

Proposition 6.6. A homogeneous real hypersurface of $\boldsymbol{C} P^{n}$ admits a skewsymmetric $(1,1)$-tensor field $B_{\xi}$ such that

$$
\alpha\left(\nabla_{\xi} A\right) X=\alpha\left(A B_{\xi} X-B_{\xi} A X\right)+\left(1+\alpha^{2}\right)\left\{g\left(X, B_{\xi} \xi\right) \xi-g(X, \xi) B_{\xi} \xi\right\}
$$

for any vector fields $X$ tangent to $M$.

We note that in particular for a homogeneous one of type $\left(A_{1}\right)$ and $\left(A_{2}\right)$, there exists a skew-symmetric $(1,1)$-tensor field $B_{\xi}=\varphi$ such that

$$
\nabla_{\xi} A=A \circ \varphi-\varphi \circ A(=0) .
$$

(See [12] and [16]). Thus we are motivated to prove the following

PROPOSITION 6.7. Let $M$ be a real hypersurface of $C P^{n}$. Suppose that $\nabla_{\xi} \xi$ 
$=0$ and $A \xi \neq-2$. If $\nabla_{\xi} A=A \circ \varphi-\varphi \circ A$, then $M$ is locally isometric to a homogeneous real hypersurface of type $\left(\mathrm{A}_{1}\right)$ and $\left(\mathrm{A}_{2}\right)$.

Proof. Using the same notations and similar calculations as in the proof of Proposition 6.5, from the rssumption we have

$$
\left(\lambda^{2}-\alpha \lambda-1\right)(\alpha+2)=0 .
$$

A similar argument as in the proof of Proposition 6.5 then yields our assertion. (Q.E.D.)

\section{References}

[1] J. Berndt and L. Vanhecke, Two natural generalizations of locally symmetric spaces, Diff. Geom. Appl. 2(1992), 57-80.

[2] - Geodesic spheres and generalizations of symmetric spaces, Boll. Un. Mat. Ital. (7), 7-A (1993), 125-134.

3 - Geodesic sprays and $(5-$ and $\mathfrak{B}$-spaces, to appear in Rend. Sem. Mat. Univ. Politec. Torino.

[4] D.E. Blair, Contact manifolds in Riemannian geometry, Lecture notes in Math. 509, Springer-Verlag, Berin-Heidelberg-New-York, 1976.

[5] D.E. Blair and L. Vanhecke, Symmetries and $\varphi$-symmetric spaces, Tôhoku Math. J. 39 (1987), 373-383.

[6] New characterizations of $\varphi$-symmetric spaces, Kodai Math. J. 10 (1987), 102-107.

[7] T.E. Cecil and P.J. Ryan, Focal sets and real hypersurfaces in complex projective space, Trans. Amer. Math. Soc. 269 (1982), 481-499.

[8] J.T. Cho, Natural generalizations of locally symmetric spaces, Indian J. Pure Appl. Math. 24(4) (1993), 231-240.

[9] A. Gray, Classification des variétés approximativement Kählériennes de courbure sectionnelle holomorphe constante, C. R. Acad. Sci. Paris Sér. A 279 (1974), 797-800.

[10] S. Helgason, Geometry and symmetric spaces, Academic Press, New York 1962.

[11] M. Kimura, Real hypersurfaces and complex submanifolds in complex projective space, Trans. Amer. Math. Soc. 296 (1986), 137-149.

[12] M. Kimura and S. Maeda, On real hypersurfaces of a complex projective space II, Tsukuba J. Math. 15 (1991), 547-561.

[13] W. Klingenberg and S. Sasaki, On the tangent sphere bundlə of a 2-sphere, Tôhoku Math. J. 27 (1975), 49-56.

[14] Y. Maeda, Oh real hypersurfaces of a complex projective space, J. Math. Soc. Japan 28 (1976), 529-540.

[15] M. Okumura, Certain almost contact hypersurfaces in Kaehlerian manifolds of constant holomorphic sectional curvatures, Tôhoku Math. J. 16 (1964), 270-284.

[16] - On some real hypersurfaces of a complex projective space, Trans. Amer. Math. Soc. 212 (1975), 355-364.

[17] S. Sasaki and Y. Hatakeyama, On differential manifolds with certain structures which are closely related to almost contact structure II, Tôhoku Math. J. 13 (1961), 282-294.

[18] R. Takagi, Real hypersurfaces in a complex projective space with constant principal 
curvatures I, II, J. Math. Soc. Japan 25 (1975), 43-53, 507-516.

[19] T. Takahashi, Sasakian $\varphi$-symmetric spaces, Tôhoku Math. J. 29 (1977), 91-113.

[20] N. Tanaka, On non-degenerate real hypersurfaces, graded Lie algebras and Cartan connections, Japan. J. Math. 2 (1976), 131-190.

[21] S. Tanno, Ricci curvature of contact Riemannian manifolds, Tôhoku Math. J. 40 (1988), 441-448.

[22] Variational problems on contact Riemannian manifolds, Trans. Amer. Math. Soc. 314 (1989), 349-379.

[23] L. Vanhecke and T.J. Willmore, Interaction of tubes and spheres, Math. Ann. 263 (1983), 31-42.

[24] Y. Watanabe, Geodesic symmetries in Sasakian locally $\varphi$-symmetric spaces, Kodai Math. J. 3 (1980), 48-55.

Department of Mathematical Science

Graduate School of Science

and Technology

Niigata University

Niigata, 950-21, Japan 